\title{
First evidences of direct interaction between 5-HT2A and $\operatorname{TrkB}$ receptors
}

\author{
Tatiana Ilchibaeva \\ ICG SB RAS, Novosibirsk, Russia \\ rbicehok@mail.ru
}

\author{
Anton Tsybko \\ ICG SB RAS, Novosibirsk, Russia \\ antoncybko@mail.ru
}

\author{
Vladimir Naumenko \\ ICG SB RAS, Novosibirsk, Russia \\ naumenko2002@mail.ru
}

\begin{abstract}
The existence of 5-HT2A-TrkB heteroreceptor complexes both in vitro and in vivo was shown for the first time. Also was found that the chronic activation of 5-HT2A receptor with agonists has decreased the total as well as membrane TrkB protein level indicating downregulation of TrkB receptors.
\end{abstract}

Keywords - 5-HT2A, TrkB, agonists, DOI, TCB-2, 25CN$\mathrm{NBOH}$, co-immunoprecipitation, expression, heterodimerization

Motivation and aim

\section{Motivation}

Cross-talk between the brain-derived neurotrophic factor (BDNF) and serotonin 5-HT2A receptors was demonstrated in the number of studies [1]. At the same time an exact mechanism of modulation of BDNF system through 5-HT2A receptors is still unknown. BDNF performs its functions using tropomyosin receptor kinase $\mathrm{B}$ (TrkB). And these receptors may be one of the possible targets. The existence of a heteroreceptor complex between the 5-HT1A and FGFR1 (FGFR1 belongs to the receptor tyrosine kinase superfamily) receptors [2] suggest a possible similar complex between 5HT2A and TrkB receptors.

Aim

The aim of present study was to investigate the presence of 5-HT2A-TrkB heteroreceptor complexes in cell cultures as well as mouse brain tissues.

\section{Methods}

Adult males of $\mathrm{C} 57 \mathrm{Bl} / 6$ mice were treated with mixed 5HT2A/2C agonist DOI or selective 5-HT2A agonists TCB-2 or $25 \mathrm{CN}-\mathrm{NBOH}$ ( $1 \mathrm{mg} / \mathrm{kg}$, i.p., 14 days). Expression of TrkB receptors was assessed by real-time RT-PCR and Western blot analysis in the frontal cortex, hippocampus, midbrain and striatum. Murine 5-HT2A cDNA was cloned into the pcDNA3.1(+) donor plasmid (Invitrogen) with HA-tag. TrkB mEGFP was a gift from Ryohei Yasuda (Addgene plasmid \#83952; http://n2t.net/addgene:83952; RRID: Addgene 83952). Mouse N1E-115 neuroblastoma cells from the American Type Culture collection (ATCC) were grown in Dulbecco's modified Eagle's medium (DMEM) containing $10 \%$ fetal bovine serum (FBS) and $1 \%$ penicillin/streptomycin at $37{ }^{\circ} \mathrm{C}$ under $5 \% \mathrm{CO} 2$. Co-immunoprecipitation and immunoblotting in N1E-115 cells co-expressing HAtag_HT2A and TrkB_mEGFP receptors were performed as described [3]. Co-immunoprecipitation from brain homogenates was performed according to [4] with modification.

\section{Results}

TrkB mRNA level was reduced after $25 \mathrm{CN}-\mathrm{NBOH}$ in the midbrain and was elevated after TCB-2 in the striatum. All of used drugs significantly decreased the TrkB total protein level in the midbrain and membrane TrkB protein level in the midbrain and striatum as well. This looks like desensitization of TrkB receptors. Using co-transfection of NIE-115 cells with 5-HT2A and TrkB receptors followed by coimmunoprecipitation the presence of heteroreceptor complexes was shown. Later using co-immunoprecipitation we have found the presence of 5-HT2A-TrkB heteroreceptor complexes in the midbrain and striatum of intact mice that was agreement with the data from chronic 5-HT2A receptor activation. Thus, for the first time the heterodimerization between 5-HT2A and TrkB receptors was found. This phenomenon might underlie the downregulation of TrkB receptors after chronic activation of 5-HT2A receptor with agonists.

\section{ACKNOWLEDGMENT}

The work supported by the RFBR (\#20-04-00253). The cost of animal maintenance was supported by the basic research project No 0324-2019-0041-C-01.

\section{REFERENCES}

[1] Jaggar M, Vaidya VA (2018) 5- $\mathrm{HT}_{2 \mathrm{~A}}$ Receptors and BDNF Regulation: Implications for Psychopathology. In: Guiard BP, Di Giovanni G (eds) 5-HT $\mathrm{HA}_{2 \mathrm{~A}}$ Receptors in the Central Nervous System, The Receptors 32. Humana Press, pp 395-438.

[2] Borroto-Escuela D.O., Tarakanov A.O., Fuxe K. (2016) FGFR1-5HT1A Heteroreceptor Complexes: Implications for Understanding and Treating Major Depression. Trends Neurosci. 39:5-15.

[3] Kobe, F., Guseva, D., Jensen, T. P., Wirth, A., Renner, U., Hess, D., Müller, M., Medrihan, L., Zhang, W., Zhang, M., et al. (2012). 5HT7R/G12 signaling regulates neuronal morphology and function in an age-dependent manner. J. Neurosci. 32, 2915-2930.

[4] Bijata M, Labus J, Guseva D, Stawarski M, Butzlaff M, Dzwonek J, Schneeberg J, Böhm K, Michaluk P, Rusakov DA, Dityatev A, Wilczyński G, Wlodarczyk J, Ponimaskin E. Synaptic Remodeling Depends on Signaling between Serotonin Receptors and the Extracellular Matrix. Cell Rep. 2017 May 30;19(9):1767-1782. 